\title{
The Underexplored Frontier of Ice Giant Interiors and Dynamos
}

A white paper for the 2023-2032 Decadal Survey on Planetary Science and Astrobiology

\section{Principal Author:}

Krista M. Soderlund, 218-349-3006, Institute for Geophysics, Jackson School of Geosciences, The University of Texas at Austin, krista@,ig.utexas.edu

\section{Co-authors:}

Mandy Bethkenhagen ${ }^{1}$, Imke de Pater $^{2}$, Jonathan Fortney ${ }^{3}$, Sebastien Hamel ${ }^{4}$, Ravit Helled ${ }^{5}$, Yong-Jae Kim ${ }^{4}$, Marius Millot ${ }^{4}$ and Sabine Stanley ${ }^{6}$

\section{Endorsements:}

Ashna Aggarwal ${ }^{7}$, Sushil Atreya ${ }^{8}$, Jonathan Aurnou ${ }^{7}$, Elizabeth Bailey ${ }^{3}$, Chloe Beddingfield ${ }^{9,10}$, Michel Blanc ${ }^{11}$, Shawn Brueshaber ${ }^{12}$, Michael Calkins ${ }^{13}$, Hao Cao ${ }^{14}$, Ian Cohen ${ }^{15}$, Federica Coppari $^{4}$, Wieland Dietrich ${ }^{16}$, Nicholas Featherstone ${ }^{13}$, Yingwei Fei ${ }^{17}$, Leigh Fletcher ${ }^{18}$, A. James Friedson $^{12}$, Alexander Goncharov ${ }^{17}$, Candice Hansen ${ }^{19}$, Emily K. Hawkins ${ }^{20}$, Matthew Hedman ${ }^{21}$, Dustin Hill $^{22}$, Christopher Hirata ${ }^{23}$, Mark Hofstadter ${ }^{12}$, Richard Holme ${ }^{24}$, George Hospodarksy ${ }^{25}$, Noam Izenberg $^{15}$, Baptiste Journaux ${ }^{26}$, Yohai Kaspi ${ }^{27}$, Peter Kollmann ${ }^{15}$, Liming Li $^{28}$, Kathleen Mandt $^{15}$, Adam Masters ${ }^{29}$, Paulo Montero-Camacho ${ }^{30}$, Kimberly Moore ${ }^{31}$, Sarah E. Moran ${ }^{6}$, Nadine Nettelmann ${ }^{32}$, Takuo Okuchi ${ }^{33}$, Norimasa Ozaki ${ }^{34}$, Marzia Parisi ${ }^{12}$, Carol Paty ${ }^{35}$, Raymond Pierrehumbert ${ }^{36}$, Lynnae C. Quick ${ }^{37}$, Bruce Remington ${ }^{4}$, James Roberts ${ }^{15}$, Leslie Rogers $^{38}$, Tamara Rogers ${ }^{19,39}$, Abigail Rymer ${ }^{15}$, Ashkan Salamat ${ }^{40}$, Didier Saumon ${ }^{41}$, Gerald Schubert $^{7}$, François Soubiran ${ }^{42}$, Linda Spilker ${ }^{12}$, Sarah T. Stewart ${ }^{43}$, Paolo Tortora ${ }^{44}$, Jake D. Turner $^{45}$, Elizabeth P. Turtle ${ }^{15}$, Jun Tsuchiya ${ }^{46}$, Taku Tsuchiya ${ }^{46}$, Johannes Wicht ${ }^{16}$, Rakesh K. Yadav $^{14}$

${ }^{1}$ Ecole Normale Supérieure de Lyon, France, ${ }^{2}$ University of California, Berkeley, ${ }^{3}$ University of California, Santa Cruz, ${ }^{4}$ Lawrence Livermore National Laboratory, ${ }^{5}$ University of Zürich, Switzerland, ${ }^{6}$ Johns Hopkins University, ${ }^{7}$ University of California Los Angeles, ${ }^{8}$ University of Michigan Ann Arbor, ${ }^{9}$ SETI Institute, ${ }^{10}$ NASA Ames, ${ }^{11}$ IRAP, CNRS-University Toulouse III CNES, France, ${ }^{12}$ Jet Propulsion Laboratory ${ }^{13}$ University of Colorado Boulder, ${ }^{14}$ Harvard University, ${ }^{15}$ Johns Hopkins University Applied Physics Laboratory, ${ }^{16}$ Max Planck Institute for Solar System Research, Göttingen, Germany, ${ }^{17}$ Carnegie Institution for Science, ${ }^{18}$ University of Leicester, UK, ${ }^{19}$ Planetary Science Institute, ${ }^{20}$ Loyola Marymount University, ${ }^{21}$ University of Idaho, ${ }^{22}$ Drexel University, ${ }^{23}$ The Ohio State University, ${ }^{24}$ University of Liverpool, UK, ${ }^{25}$ University of Iowa, ${ }^{26}$ University of Washington, ${ }^{27}$ Weizmann Institute of Science, Israel, ${ }^{28}$ University of Houston, ${ }^{29}$ Imperial College London, UK, ${ }^{30}$ Tsinghua University, Beijing, China,

${ }^{31}$ California Institute of Technology, ${ }^{32}$ German Aerospace Center, Germany, ${ }^{33}$ Okayama University, Japan, ${ }^{34}$ Osaka University, Japan, ${ }^{35}$ University of Oregon, ${ }^{36}$ University of Oxford, UK, ${ }^{37}$ NASA Goddard Space Flight Center, ${ }^{38}$ University of Chicago, ${ }^{39}$ Newcastle University UK, ${ }^{40}$ University of Nevada, Las Vegas, ${ }^{41}$ Los Alamos National Laboratory, ${ }^{42}$ CEA DAM-DIF, France, ${ }^{43}$ University California Davis, ${ }^{44}$ University of Bologna, Italy, ${ }^{45}$ Cornell University, ${ }^{46}$ Ehime University, Japan

Part of this work was performed by Lawrence Livermore National Laboratory under Contract DE-AC52-07NA27344. 


\section{Introduction}

The interiors of Uranus and Neptune play a critical role in almost every aspect of their systems, ranging from bulk composition and heat flow to the dynamo and atmospheric dynamics to the configuration of the magnetosphere and potential resonant oscillations in the rings. Bulk composition of the planets, especially noble gas abundances and isotopic ratios of $\mathrm{H}, \mathrm{C}, \mathrm{N}, \mathrm{S}$, and $\mathrm{O}$, is also key to how the giant planets formed and evolved ${ }^{[1]}$. The planetary dynamo was a high priority science objective of the Visions \& Voyages Decadal Survey, and broader Interior science has become even more prioritized over the past decade. The major revelations of the structure and mechanics of the interiors of Jupiter and Saturn from Juno and the Cassini Grand Finale missions, respectively, place our ignorance of ice giant interiors in stark contrast ${ }^{[2,3]}$. Furthermore, the discovery from the Kepler Mission of vast numbers of planets from 2 to 4 Earth radii $^{[4]}$, often called "mini-Neptunes", gives additional impetus to understanding the interiors and dynamos of Uranus and Neptune.

\section{State of the Science: Internal Structure}

It is remarkable how little is known with much certainty about the interiors of Uranus and Neptune. Questions of the bulk composition, interior density and temperature structure, and thermal evolution history have long remained opened, and with currently available data there is no clear path towards resolving these issues.

Formation scenarios for the planets find it difficult to explain that $10-20 \%$ of the planetary mass is in the $\mathrm{H} / \mathrm{He}$ envelope, without considerable fine tuning ${ }^{[5]}$. It is hard to form Uranus and Neptune in a few million years and keep them from becoming much larger gas giants. In detail, there is a greater appreciation now that accretion of solids and $\mathrm{H} / \mathrm{He}$ during formation likely leads to composition gradients in the $\mathrm{H} / \mathrm{He}$ envelope ${ }^{[6]}$, but it is not yet clear to what degree these gradients last, due to convection ${ }^{[7]}$. Uranus and Neptune could well have diverged in their history early on due to giant impacts radically altering interior composition gradients and temperature structures $^{[8]}$.

Textbook models of the planets show a predominantly $\mathrm{H} / \mathrm{He}$ convective envelope atop a thick water-dominated fluid convective mantle layer, with a rocky core. Models like these can be constructed that match either planet's relatively poorly-known gravity field. However, such models have never yielded adequate fits to the planetary heat flow governed by thermal evolution $^{[9]}$. An extremely wide range of interior models can actually fit the gravity fields, including those with no water at all ${ }^{[10]}$.

The past decade has seen significant advances in understanding the unusual properties of water, ammonia, methane, and their mixtures when submitted to the high-pressure, high-temperature conditions expected inside icy planets, using first-principles theory as well as experiments ${ }^{[11,12]}$. Interior views like Figure 1 suggest a high degree of specific knowledge about the current structure, but this is really an illusion. A large number of assumptions have been made to construct such a model, including the current temperature structure (adiabatic, or super-adiabatic to an unknown degree), the relative ratio of $\mathrm{C} / \mathrm{N} / \mathrm{O}$ that gives rise to methane, ammonia, and water, and whether or not there is distinct layering. The long-standing problem with this kind of state-of-the art models shown below is that they typically yield ice-to-rock ratios of 3-to-1 to 20 -to-1, which is incredibly high ${ }^{[13]}$. For reference, Pluto is thought to be $70 \%$ rock, so it is unclear how such outer solar system giant planets could form so ice-rich. Alternative structure models posit mimicking the density of ices with mixtures of $\mathrm{H} / \mathrm{He}$ and rock, 
in a layered scenario, which helps to alleviate the ice/rock ratio problem ${ }^{[10]}$, but then opens up a wide phase space to possible formation and evolutionary histories.

Convenient 3-layer adiabatic models for Uranus and Neptune are also computationally easy to run thermal evolution calculations for, and much work has gone into trying to get such models to fit the Voyager-era energy balance data. Advances in material properties at planetary interior conditions have changed the specifics of evolutionary models over time, although the reality is that there is no adequate thermal history calculation for either planet. Historically, Uranus has been substantially under-luminous, and Neptune modestly so ${ }^{[14]}$. However, recent work alters this view, with Uranus being modestly under-luminous and Neptune being over-luminous, compared to 3-layer-adiabatic models ${ }^{[15]}$. The recent dramatic reassessment of Jupiter's energy balance ${ }^{[16]}$ from Cassini flyby data, compared to Voyager data, also brings the evolutionary stories developed in the past decade for Uranus and Neptune based on Voyager data into question, so it is critical for the energy balance of both planets to be assessed anew.
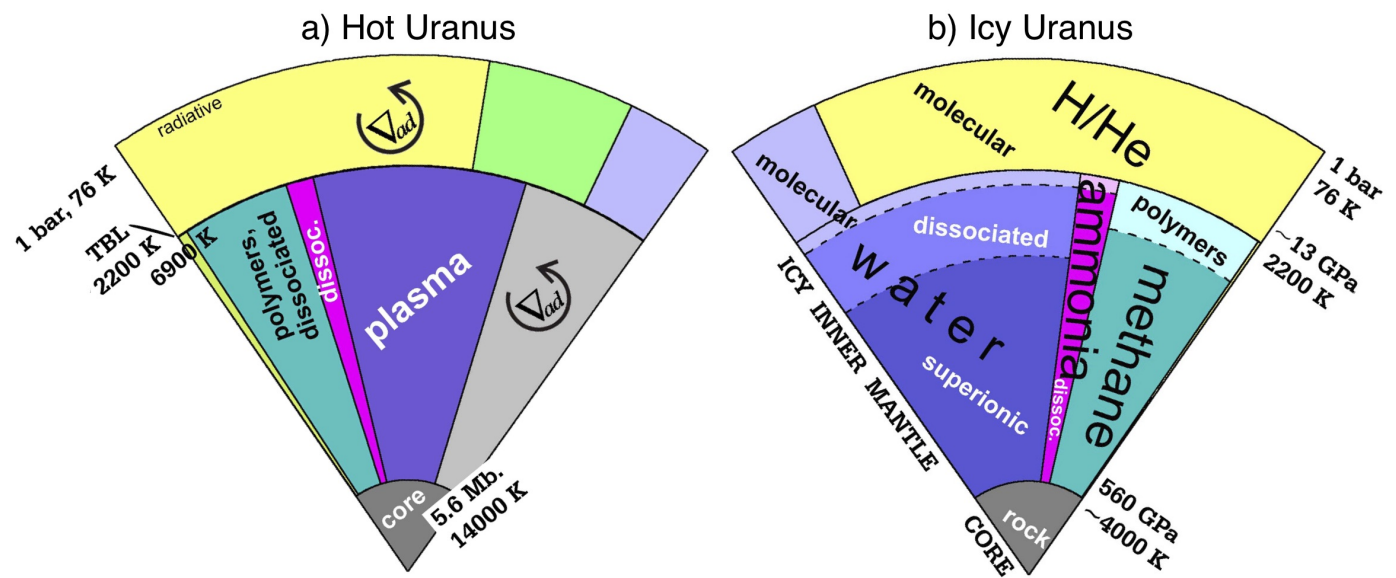

Figure 1: The internal structures of Uranus (and Neptune) are poorly constrained, and no models at present are able to satisfy the gravity, magnetic, and heat flow observations simultaneously without ad-hoc assumptions. Two three-layer internal structure model examples for Uranus. a) 'Hot Uranus' model with an imposed thermal boundary layer (TBL) that fits gravity and luminosity data [17]. b) 'Icy Uranus' model without a TBL using updated equations of state that fits gravity but not luminosity data [11].

Additional factors that come into play in interior structure modeling include the heavy element enrichment of the $\mathrm{H} / \mathrm{He}$ envelope, which could (in part) be assessed via an entry probe. The rotation periods of both planets, a critical ingredient in structure models, are likely more uncertain than the typical stated error bars ${ }^{[18]}$. The degree to which winds in the visible atmosphere extend into the deeper interior has been a breakthrough discovery for Jupiter and Saturn in the past few years ${ }^{[19,20]}$, and there is already some evidence for a quite dissimilar, shallower depth in Uranus and Neptune ${ }^{[21]}$ which could be greatly refined.

3. State of the Science: Dynamo

As the solar system has been explored with spacecraft missions, our understanding of planetary magnetic fields has developed from the Earth as an archetypal example to learning about the large breadth of behaviors exhibited from Mercury to Neptune (Fig. 2) ${ }^{[22]}$. Earth and Jupiter have dipole-dominated fields with $\sim 10^{\circ}$ tilts, prominent regions of enhanced intensities, and measured secular variation, although with surface intensities that differ by more than an order of magnitude. In contrast, Mercury and Saturn have strikingly axisymmetric fields, with varying 
degrees of equatorial asymmetry between the northern and southern hemispheres, and slow secular variation; surface field strengths here differ by nearly two orders of magnitude. Uranus and Neptune have multipolar fields with comparable intensities and no clear symmetries along any axis; no information about secular variations is known at present. These three categories lead to overarching questions about planetary magnetic field generation: What processes control magnetic field morphology, strength, and temporal evolution? What aspects of the planetary interiors are responsible for the variations we see across the terrestrial, gas giant, and ice giant planets as well as within each of these classes? The ice giants thus provide an ideal laboratory to test dynamo hypotheses.

a) Mercury $(\ell \leq 5)$

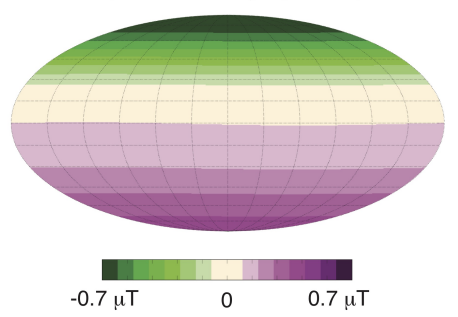

b) Earth $(\ell \leq 13)$

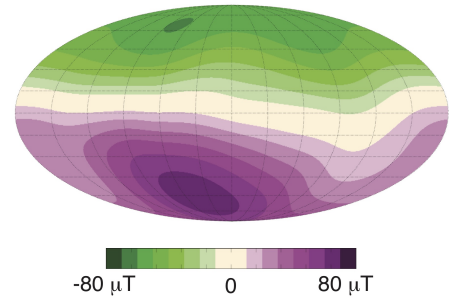

c) Jupiter $(\ell \leq 10)$

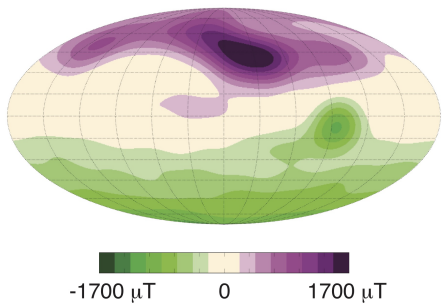

d) Saturn $(\ell \leq 11)$

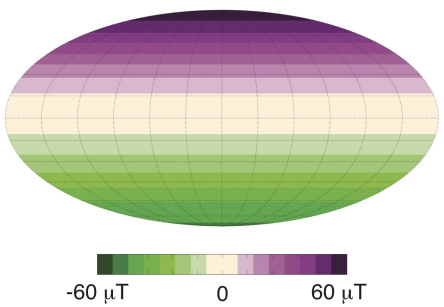

e) Uranus $(\ell \leq 4)$

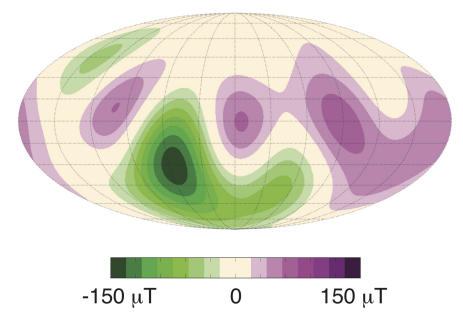

f) Neptune $(\ell \leq 3)$

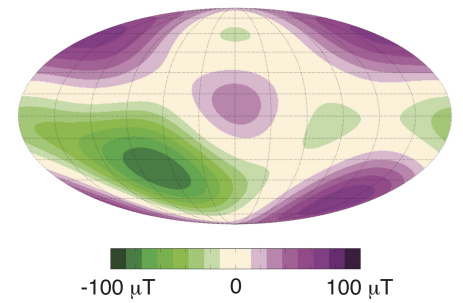

Figure 2: The magnetic fields of Uranus and Neptune are fundamentally different from all known planetary dynamos, illustrated by their radial fields at the surface [22]. Because of the limited spatial resolution (given by spherical harmonic degree $\ell$ ) at the ice giants, their fields are likely to be considerably more detailed than existing observations reveal. Purple (green) indicates outward (inward) directed field.

The lack of strong constraints on the ice giants' interior structure, composition, and thermal profiles limits our ability to determine exactly where inside Uranus and Neptune the dynamos are generated. With present data and constraints, we are forced to use a combination of elimination and plausibility to answer this question. The hydrogen/helium envelope does not reach pressures high enough to attain strong electrical conductivity, while the rocky core is likely too deep to explain the multipolar nature of the surface magnetic fields. Eliminating these two layers leaves the ice-rich mantle layer. Considering water as an example of the ices making up the majority of Uranus and Neptune, the phase diagram suggests that appreciable conductivity can be reached at depths of 0.2-0.3 planetary radii below the surface in Uranus and Neptune.

Although convection in the deep interior is widely agreed as the source of the ice giants' magnetic fields, the underlying reason why the fields are multipolar and non-axisymmetric remains poorly understood. Two possible mechanisms for generating ice giant-like magnetic fields ${ }^{[23]}$ include: a solid inner core that is less electrically conductive than the overlying fluid shell or a deep stably-stratified layer beneath a convecting fluid shell. Although the properties of superionic ice are not well known, it is believed to behave as a solid ${ }^{[12]}$ and have an electrical 
conductivity exceeding that of ionic water ${ }^{[24]}$, arguing against the first option. While the presence of a superionic ice layer would also argue against the second option, properties of the interior are sufficiently poorly constrained that stable stratification in the deep interior cannot be ruled out ${ }^{[25]}$. An alternative hypothesis is that the ice giants have multipolar dynamos because convective turbulence in their interiors is weakly constrained by rotation (i.e. inertial effects are strong), in contrast to rotationally constrained convection in the deep interiors of the gas giants that drives dipole-dominated dynamos ${ }^{[26-28]}$. While numerical dynamo models show this convective regime leads to magnetic fields, zonal winds, and internal heat flux patterns that agree qualitatively with observations $^{[27]}$, it is not yet clear if weakly constrained convection is likely in the ice giant interiors. Other potential explanations include bistability ${ }^{[29,30]}$ and the interplay between density and electrical conductivity variations with radius ${ }^{[31]}$. In order to determine which-if any-of these explanations is correct, additional work is needed in regards to observational constraints, physical properties of ice giant interiors, and dynamo and rotating convection behavior as described in the next section.

\section{Key Science Questions}

Many questions remain about Uranus' and Neptune's interiors and magnetic fields, such as:

1) What is the internal density and compositional distribution? What parts of each planet are adiabatic?

2) How deep do the atmospheric circulations observed on the surface extend into the interior and do they interact with the dynamo?

3) How do the thermodynamic and transport properties of the planets vary with radius and with time as the planet evolves?

4) What are the detailed configurations of their magnetic fields? Has secular variation occurred since the Voyager 2 flybys?

5) What processes generate the dynamo? What are the characteristics of zonal winds, meridional circulations, and turbulent convective flows in the deep interior?

6) What are the internal structures and dynamo characteristics of ice giant exoplanets? How do they compare to Uranus and Neptune, as well as to gas giant exoplanets and super-Earths?

Questions 1, 2, and 4 illustrate the need for a new mission to Uranus and/or Neptune ${ }^{\text {[WP1-2]. }}$ Question 3 relies on advancements in planetary materials at high pressures and temperatures, coupled with internal structure models. Continued numerical and laboratory experiments of convection and dynamo action are necessary for Question 5. Comparative planetology is another powerful tool to understand ice giant planets, per Question 6.

Question 1: What is the internal density and compositional distribution? What parts of each planet are adiabatic? The gravitational field of Uranus is $\sim 100$ times more poorly known than for Jupiter and Saturn, and for Neptune this is a factor of $\sim 1000$. The combination of gravitational field measurements and detailed interior models is our traditional window into the interior density distribution. A Uranus or Neptune orbiter that includes some close-periapse orbits can dramatically enhance our view of either planet's density structure. An entry probe to measure the abundance of volatile gasses would further constrain $\mathrm{H} / \mathrm{He}$ envelope metallicities.

Assessing any (thought to be likely) deviations from adiabaticity are likely more difficult. Comprehensive work that ties formation to thermal evolution, with the best available material property models, can shed light on interior temperature distributions for models that also match the gravitational field. Essential complementary work would firmly establish the internal heat flow $^{[\mathrm{WP} 3]}$. The most promising route for more direct assessments of temperature structure would 
come from seismology ${ }^{[32]}$, either from the direct detection of oscillations (from the ground or from space, perhaps in orbit) or potentially from ring seismology, the prospects for which should be more actively investigated.

Question 2: How deep do the atmospheric circulations observed on the surface extend into the interior and do they interact with the dynamo? The depth of zonal wind penetration into the planets may be established through precise measurements of the high-order gravity field, which preferentially probes planetary depths closer to the surface. Such new work was some of the most celebrated science of the Juno and Cassini Grand Finale Missions. This higher order field can only be accessed via low-periapse orbits within $\sim 1.1$ planetary radii. Microwave and radio sounding of the planet would further constrain flows across the troposphere ${ }^{[\mathrm{WP} 4]}$. Observations of planetary oscillations might further constrain interior flows and identify layers of convection versus stable stratification. In-situ measurements of winds, temperatures, and composition by a probe would provide ground truth for interpretations.

Question 3: How do the thermodynamic and transport properties of the planets vary with radius and with time as the planet evolves? Our understanding of the interior structure, formation, and evolution of these massive planets is limited by our understanding of how the extreme pressure-temperature conditions expected in the deep interior affect the properties of the constituent material. The equation of state (EOS) is one of the most important modeling inputs. By this we mean an EOS that captures the essential impact of chemistry at high pressure and that properly treats multi-phase and multi-component fluids and solids over a wide range of temperatures and pressures. The dynamics of convecting regions additionally depends on transport properties (e.g., viscosity, electrical and thermal conductivities) of these materials that ultimately control convection and dynamo action, and their variations with depth may also modulate magnetic field generation.

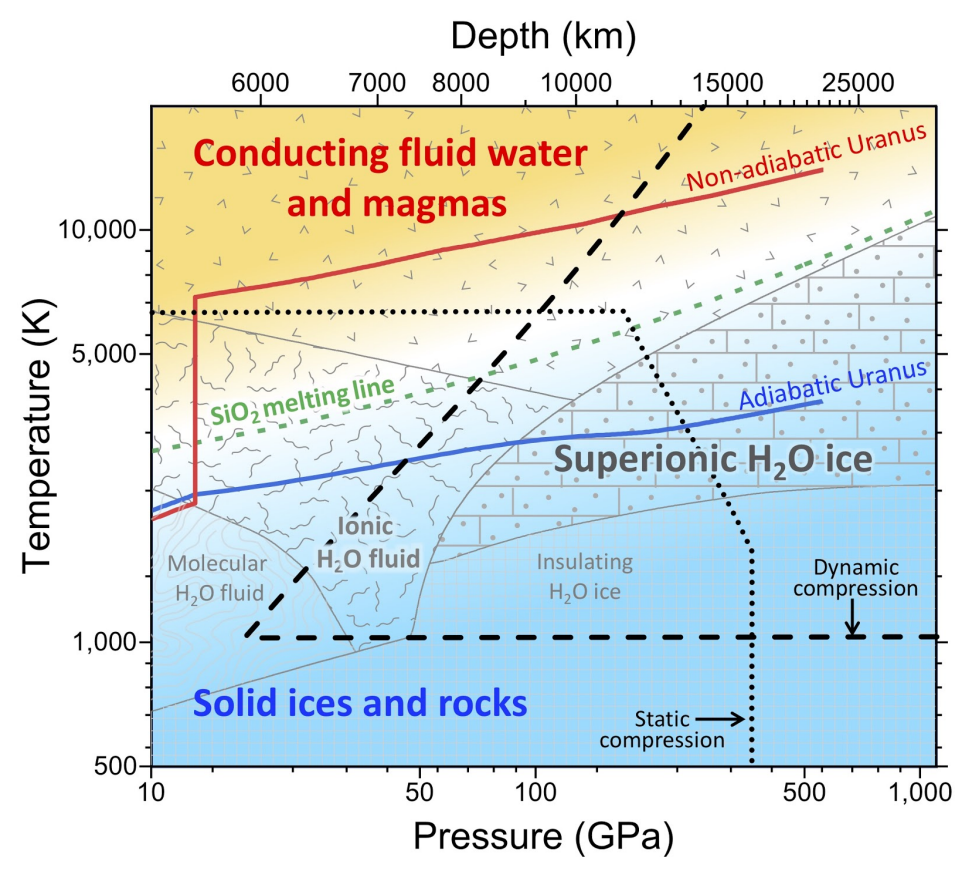

Figure 3: Laboratory experiments and numerical simulations reveal new material behaviors within ice giant interiors. Example profiles of Uranus' mantle include a hot non-adiabatic model [red line, 17] and a cold adiabatic model [blue line, 11]. The phase diagram of water [gray line, 12] and the melting line of silica [green line, 33], showing the conducting fluid water and magmas and the solid ices and rocks (yellow and blue shaded regions). Range of static and dynamic compression experiments (black dotted and dash lines). Quantum simulations can complement experiments and map material properties over the full range of PT conditions shown here.

Advances in experimental techniques and in quantum electronic structure simulations now allow us to study the effects of megabar pressures and thousands of degree temperatures on the microscopic, thermodynamic and transport properties of icy planets materials. For example, 
Fig. 3 overlays accessible conditions with either static or dynamic compression experiments with the simplified phase diagram of water and silica as prototypical ice and rock materials. In contrast with their behavior at the surface of the Earth, new behaviors emerge at depth within the icy giants, such as superionic ices, ionic and conducting fluids, and conducting magmas. Experimental and computational advances are expected to continue in the upcoming decades, in particular towards a better grasp on mixtures and multi-phase phenomena.

Question 4: What are the detailed configurations of their magnetic fields? Has secular variation occurred since the Voyager 2 flybys? New magnetic field measurements close to the planet at a variety of latitudes and longitudes would allow characterization of the ice giants' higher degree magnetic field structure, ideally to better than spherical harmonic degree 10 for comparison with Earth, Jupiter, and Saturn and also to estimate the top of the dynamo region $^{[34]}$. This field determination could be further improved through imaging of auroral and satellite footprints that provide additional high-latitude constraints. Observations of the distributions of energetic charged particles that follow the magnetic field lines allow constraining the non-local structure of the field. The internal magnetic field may have undergone temporal change since the Voyager 2 epoch so new observations, even from a flyby, would provide constraints on secular variation and potentially identify changes in the locations of flux patches that are indicative of zonal and/or meridional winds in the deep interior ${ }^{[35]}$.

Question 5: What processes generate the dynamo? What are the characteristics of zonal winds, meridional circulations, and turbulent convective flows in the deep interior? As new hypotheses for magnetic field generation progress with advancements in ice giant internal structures and physical properties, dynamo models will provide a means to test these ideas. In order to better understand interior-atmosphere interactions, coupling of dynamo models with atmospheric dynamics and radiative transfer models would also be a fruitful future direction. These models would make detailed predictions about magnetic fields, flow fields, and heat transfer that could serve to guide instrument and mission concept designs. Additionally, it is important to better understand and test convective regime transitions and their influence on magnetic field generation to answer questions like the feasibility of the ice giants being in the weakly rotating convective regime. As a last example, more realistic parameters should be investigated as computing capabilities continue to improve with time since viscous effects may be (artificially) important in existing models ${ }^{[36]}$ and novel behaviors are being found as new regions of parameter space are explored ${ }^{[37]}$.

Question 6: What are the interior and dynamo characteristics of ice giant exoplanets? How do they compare to Uranus and Neptune, as well as to gas giant exoplanets and super-Earths? It is now clear from transiting planet bulk densities, that lower mass giant planets from $\sim 15 \mathrm{M}_{\text {Earth }}$ to $\sim 10 \mathrm{M}_{\text {Jupiter }}$ are much more enriched in heavy elements ${ }^{[38]}$, a pattern seen with a sample size of 4 in our solar system. In the TESS era, the statistical characterization of a much larger number of planets will lead to even better constraints. Complementing this work, JWST (launching early in the 2020s) and ARIEL (scheduled for 2028) will establish atmospheric enrichments at least for C, N, O-bearing molecules, and likely several others, as a function of planetary mass. An entry probe into Uranus or Neptune would provide complementary information to both Jupiter and the exoplanet population.

Exoplanetary magnetic fields have recently been detected (indirectly) for the first time ${ }^{[39]}$. Determining whether these planets have dipolar or multipolar dynamos and their zonal wind profiles would provide critical data points to assess dynamo generation hypotheses more 
broadly ${ }^{[40]}$. As we learn more about ice giant planets around other stars, we will also learn more about Uranus and Neptune closer to home.

\section{Recommendations}

We make the following recommendations for the panel to consider:

- Prioritize a new orbiter mission to Uranus and/or Neptune that includes interior science of the planets, with gravity and magnetic field measurements being most important. These measurements should be taken close to the planet $(<1.1$ planetary radii) and have good latitude-longitude spatial coverage. Energy balance measurements are also critical.

- Advise that multidisciplinary laboratory, simulation, and theoretical studies are necessary for advances in understanding the interiors and dynamos of ice giant planets. Advocate for a robust Research \& Analysis budget and continued investment in High End Computing capabilities.

- Form a network for giant planet studies to facilitate cross-disciplinary collaboration and discoveries, similar to programs such as the Interdisciplinary Consortia for Astrobiology Research (ICAR).

- Promote the diversity, inclusion, and equity of under-represented minorities in the planetary science community to achieve the best science by fostering a welcoming environment for all.

\section{References and White Papers}

[1] Mousis, O., et al. PSS 155 (2018): 12-40. [2] Cao, H., et al. Icarus (2019): 113541. [3] Stevenson, D. AREPS 48 (2020). [4] Borucki, W.J. PAPS 161.1 (2017): 38. [5] Frelikh, R. \& R. Murray-Clay. Astron. J. 154.3 (2017): 98. [6] Helled, R. \& D. Stevenson. ApJL 840.1 (2017): L4. [7] Vazan, A. \& R. Helled. $A \& A 633$ (2020): A50. [8] Reinhardt, C., et al. MNRAS 492.4 (2020): 5336-5353. [9] Fortney, J. \& N. Nettelmann. SSR 152.1-4 (2010): 423-447. [10] Helled, R., et al. ApJ 726.1 (2011): 15. [11] Bethkenhagen, M., et al. ApJ 848.1 (2017): 67. [12] Millot, M., et al. Nature 569.7755 (2019): 251-255. [13] Nettelmann, N., et al. PSS 77 (2013): 143-151. [14] Fortney, J.J., et al. ApJ 729.1 (2011): 32. [15] Scheibe, L., et al. A\&A 632 (2019): A70. [16] Li, L., et al. Nature Comm. 9.1 (2018): 1-10. [17] Nettelmann, N., et al. Icarus 275 (2016): 107-116. [18] Helled, R. et al. Icarus 210.1 (2010): 446-454. [19] Kaspi, Y., et al. Nature 555.7695 (2018): 223-226. [20] Iess, L., et al. Science 364.6445 (2019). [21] Kaspi, Y., et al. Nature 497.7449 (2013): 344-347. [22] Soderlund, K. \& Stanley, S., ESSOAr, doi: 10.1002/essoar.10503671.1. [23] Stanley, S. \& J. Bloxham. Icarus 184.2 (2006): 556-572. [24] Millot, M., et al. Nature Physics 14.3 (2018): 297-302. [25] Helled, R., et al. SSR 216.3 (2020): 1-26. [26] Aurnou, J. et al. Icarus 190.1 (2007): 110-126. [27] Soderlund, K., et al. EPSL 333 (2012): 9-20. [28] King, E. \& J. Aurnou. PNAS 110.17 (2013): 6688-6693. [29] Gastine, T., et al.. A\&A 546 (2012): A19. [30] Yadav, R., et al. Icarus 225.1 (2013): 185-193. [31] Wicht, J., et al. In Magnetic fields in the solar system. Springer, Cham, 2018. 7-81. [32] Friedson, A. Phil Trans A. doi: 10.1098/ rsta.2019.0475. [33] Millot, M., et al. (2015). Science, 347(6220), 418-420. [34] Tsang, Y.-K., \& C. Jones EPSL 530 (2020): 115879. [35] Moore, K., et al. Nature Astron. 3.8 (2019): 730-735. [36] Soderlund, K., et al. EPSL 333 (2012): 9-20. [37] Aurnou, J., et al.. PEPI 246 (2015): 52-71. [38] Thorngren, D., et al. ApJ 831.1 (2016): 64. [39] Cauley, P., et al. Nature Astron. 3.12 (2019): 1128-1134. [40] Tian, B. \& S. Stanley. ApJ 768.2 (2013): 156. [WP1] Beddingfield, Li, et al. Exploration of the Ice Giant Systems. [WP2] Cohen et al. New Frontiers-class Uranus Orbiter: Exploring the feasibility of achieving multidisciplinary science with a mid-scale mission. [WP3] Li et al. Radiant Energy Budgets and Internal Heat of Planets and Moons. [WP4] de Pater et al. Prospects to study the Ice Giants with the ngVLA. 